\title{
Practical works in the speckle optics for the subspecialties: physics of laser measurements and biomedical optics
}

Sergey Ulyanov, Sergey Kuzmin, Andrey Bednov, Valery Tuchin

Sergey S. Ulyanov, Sergey Yu. Kuzmin, Andrey A. Bednov, Valery V. Tuchin, "Practical works in the speckle optics for the subspecialties: physics of laser measurements and biomedical optics," Proc. SPIE 2525, 1995 International Conference on Education in Optics, (13 October 1995); doi: $10.1117 / 12.224057$

Event: SPIE's 1995 International Symposium on Optical Science, Engineering, and Instrumentation, 1995, San Diego, CA, United States 


\title{
Practical works in the Speckle Optics for the subspecialities "Physics of Laser Measurements" and "Biomedical Optics"
}

\author{
Sergey S. Ul'yanov, Sergey Y. Kuzmin, Andrey A. Bednov, Valery V. Tuchin
}

Saratov State University, Saratov, 410071, Astrakhanskaya 83, Russia

\begin{abstract}
The paper describes two practical works in Optics Laboratory for undergraduates to whom the courses on Statistical Optics, Diffraction Methods in Biomedicine and Physical Principles of Laser Measurements are taught. The first work belongs to a training course on Numerical Methods and Computing. Through it the undergraduates can analyze the regularities of speckles forming at coherent beams scattering in random media. The set of educational training software enables students to model the process of strongly focused Gaussian beams and focused speckle fields scattering from moving phase screen and to investigate the statistical characteristics of scattered field. Another practical work, designed on the base of two-coordinate robot with aerodynamic suspension and a linear pitch drive, is a scanning laser profilometer used for an analysis of the first and second order statistics of statistically inhomogeneous speckles and for examining the profilometer's output signal as well.
\end{abstract}

Key words: scattering, speckles, statistics

\section{INTRODUCTION}

For several years Optics Department of Saratov State University provides lectures related to Speckle Optics. There are courses on Statistical Optics, Diffraction Methods in Biomedicine, Physics of Laser Measurements, Speckleinterferometry.

The teaching of the courses is accompanied by a number of practical works carried out by students in Special Laboratory of Optics. The presented paper describes only two of them, that have been developed recently. Description of another two can be found in Ref.1. The first Laboratory work is included in the set of practical works on Basics of Numerical Modeling, another one is a part of Special Laboratory on Physics of Laser Measurements.

\section{COHERENT BEAMS DIFFRACTION FROM RANDOM SURFACES}

To supply students with a useful tool for studying of the diffraction in the cases when the analytical methods cannot give satisfactory results, a software package has been developed. It allows to simulate the processes of coherent waves diffraction both with a smooth front and speckle-modulated one from surfaces with given statistical properties. This practical work includes following exercises:

\section{a. Investigation of Gaussian beams diffraction on moving random surfaces.}

The package of programs developed for students training using Borland Pascal 7.0 Language (this is one of the programming languages taught to students of the Physics Department of Saratov State University ) makes it possible on the base of scalar diffraction theory to calculate Kirchhoff integral for a coherent field scattered from a rough surface with given statistics and perform a statistical analysis of speckle field intensity fluctuations for different observation conditions and large number of the surface relief realizations. Using Kirchhoff approximation the scattered complex amplitude $U(X, Y, Z)$ in the observation plane at the distance $\mathrm{Z}$ from the plane of scattering $(\xi, \eta, 0)$ can be written as:

$$
U(X, Y, Z)=i \iint_{-\infty}^{\infty}\left(Z / R^{2}\right) \exp \left(-\frac{\xi^{2}+\eta^{2}}{W_{0}^{2}}\right) \exp (i 4 \pi H(\xi, \eta)) \exp (i 2 \pi R) d \xi d \eta
$$

where function $H(\xi, \eta)$ describes the scattering surface relief with zero mean value in the plane of scattering $(\xi, \eta), R$ is the distance between the points $(X, Y)$ and $(\xi, \eta)$ in observation and scattering plane respectively. 
Fulfilling the exercises students are to investigate with the help of provided computer programs the first- and secondorder statistics of intensity and phase fluctuations in dynamic speckles formed at Gaussian beams diffraction from rough surfaces with Gaussian and non-Gaussian statistics. Samples of distributions obtained in this work are given in Fig.1.

\section{b. Investigation of random diffraction of focused speckles}

When the speckle field diffraction on a rough surface is analyzed, the scattered field complex amplitude can be evaluated from integral :

$$
U(X, Y, Z)=i \iint_{-\infty}^{\infty}\left(Z / R^{2}\right) U(\xi, \eta) \exp (i 4 \pi H(\xi, \eta)) \exp (i 2 \pi R) d \xi d \eta,
$$

where $U(\xi, \eta)=A(\xi, \eta) \exp (i 2 \pi \Phi(\xi, \eta)), A(\xi, \eta)$ is the illuminating field amplitude, obeys to Raleigh statistics and $\Phi(\xi, \eta))$ is the phase of speckle field, uniformly distributed random value. As in previously described case, students are to analyze the 1st and 2nd order statistics of doubly scattered speckles. Several histograms obtained in this case are presented in Fig. 2.

\section{PRACTICAL WORK "LASER PROFILOMETRY FOR RANDOM SURFACES"}

The scanning laser profilometer is used for the laboratory work performance. This profilometer is. made on the base of two-coordinate robot supplied both with aero-dynamical suspension and linear pitch-drive. The optical scheme of the device is presented in Ref.1. When focuced beam is diffracted by the surface the scattered wave is spreading in space and represents a number of Gaussian beams overlaping each other when the following conditions are fulfilled:

$$
\pi W_{0} / 2 \lambda<D / \lambda,
$$

where $W_{0}$ is the Gaussian waist-beam radius, $D$ - period of the investigated grating. When the condition (3) is fulfilled the central region of diffraction picture represents the result of interference of zero-th and $\mathrm{n}$ th orders of diffraction order. When the beam moves relately to surface with harmonic relief with velocity $v$ the phase of diffraction orders changes in time according to the law:

$$
\Psi_{n}=2 \pi n t v / D+n \Psi_{0},
$$

where $\psi_{0}$ is some unimportant constant (a value of the phase for lst diffraction order in the moment $t=0$ ).

As the result of interference of diffraction order components with mirror component used as reference wave the spatial redistribution of light intensity occurs between the +1 and -1 orders. This process occuring due to the phase change of the scattered fields components oscillates in time with period $T=v / D$. The harmonic amplitudes of variable component of output signal will be described by relation of the type:

$$
I_{n}=J_{0}(4 \pi A / \lambda) J_{n}(4 \pi A / \lambda) K\left(n, w_{0} / \lambda, D / \lambda\right) \cos \left(2 \pi n t v / D+n \psi_{0}\right)
$$

where $A$ is the amplitude of the grating relief, $\mathrm{K}$ describes the decreasing, relatively to the center of thewaist-beam, of the amplitude of the field in Gaussian beam with the waist Wo when the beam deflects at angle $\Psi_{n}, J_{n}$ is the Bessel function of $\mathrm{n}$-th order.

Laboratory work consists of two exercises:

\section{a. Determination of output characteristics of the laser profilometer}

For this task the scanning with the focused Gaussian beam is performed along the surface having harmonical relief, i.e. phase grating diffraction takes place in this case. Realizations of typical output registered by the students are presented in Fig.3. The analysis of profilometer output characteristics and the way of their definition have been described in detail ${ }^{1}$. The amplitude-frequency characteristics of the system are described by function $\mathrm{K}\left(\mathrm{D} / \mathrm{W}_{0}\right)$ (Fig.4), and the amplitude characteristics by function $J_{0}(4 \pi \mathrm{A} / \lambda) J_{\mathrm{n}}(4 \pi \mathrm{A} / \lambda)$. As a rule, for the employing of the laboratory work considered the students need to define either amplitude characteristics or amplitude-frequency ones.

\section{b. Investigation of the characteristics of speckle-fields formed with a small number of scatterers}

Statistical characteristics of speckle-fields formed at the diffraction with a small number of scatterers have been explored in detail in the papers ${ }^{3,4}$, which are recommended for students as a methodical material. For the performance of the laboratory work two tasks have to be carried out by the students:

- analysis of dependence of dynamic speckle intensity fluctuation dispersion $\sigma_{I}$ on observation point coordinate;

- analysis of dependence of dynamic speckle contrast on observation point coordinate. 
The plots of dependencies registered by the students are shown in Fig.5. and Fig.6., respectively.

\section{CONCLUSIONS}

It should be noted that special courses of lectures on Statistical Optics, Diffraction Methods in Biomedicine, Physics of Laser Measurements and Speckle-interferometry mentioned in the introduction are delivered to the fourth-year students. The first laboratory work discussed in the paper is carried out by the students of the third year of studying. The work serves as a part of the practical course on mathematical modeling. Authors of the paper consider that it is useful from the methodical viewpoint. Primary knowledge received during independent laboratory work performance connected with the analysis of speckle statistics will improve the acceptability of the materials represented in the lecture course.

\section{REFERENCES}

1. AF. Rezchikov, V.P. Ryabukho, V.V. Tuchin, S.S. Ul'yanov. Measurements of Surface Microprofile Using Diffraction of Strongly Focused Laser Beams. Problems of Machinery and Reliability of Machines, N1, 1993, pp.125-131. .

2. S.S. Ul'yanov. Analysis of the surfaces with complex periodical relief. Opt. Eng. Bulletin, NI(5), 1995, pp.20-23.

3. E. Jakeman.Speckle statistics with a small number of scatterers, Opt. Eng., Vol.23,pp. 453-461, 1984.

4. S.S. Ulyanov, D.A. Zimnyakov, V.V. Tuchin. Fundamentals and applications of dynamic speckles induced by focused laser beam scattering. Optical Engineering, Vol.33, N10, pp.3189-1994.

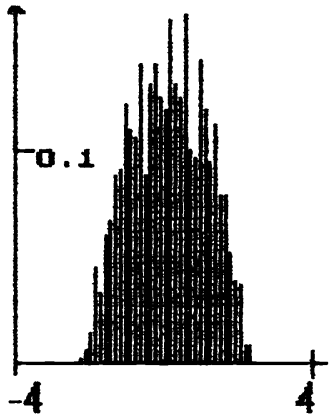

a

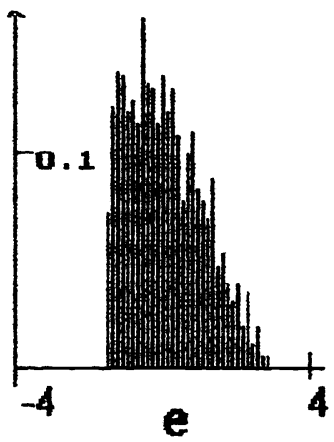

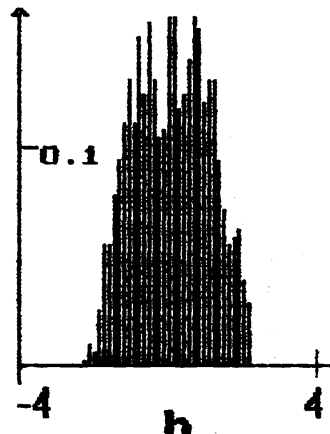

b

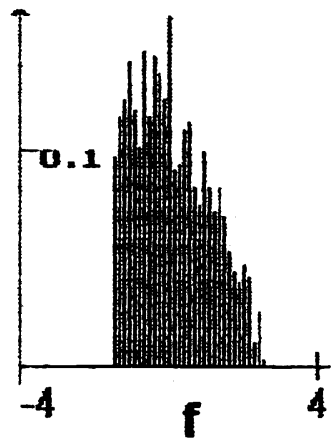

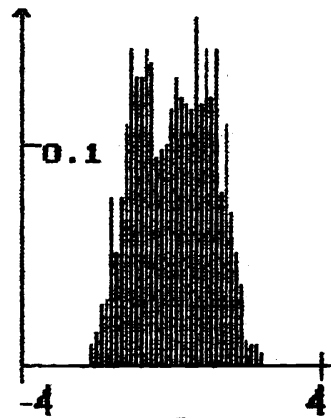

C

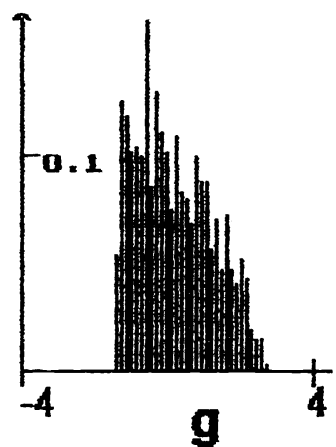

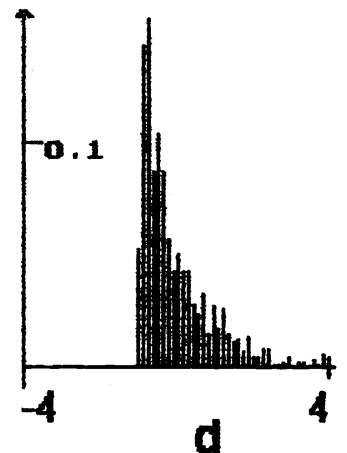

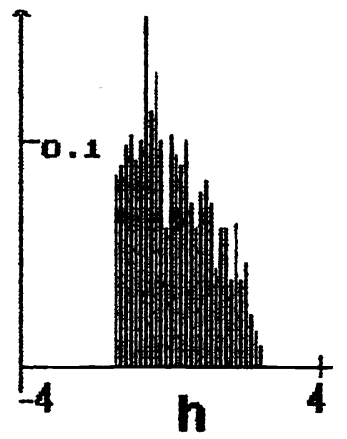

Fig. 1. Probability density function of speckles intensity fluctuations at focused Gaussian beam scattering. Values of intensity are normalized to that ones with zero mean and the unit standard deviation. Edge of diffraction pattern.

$$
\begin{array}{ll}
\sigma_{\mathrm{S}}=0.001: \text { a) } \mathrm{W}_{0} / \mathrm{L}_{\mathrm{C}}=0.5 ; \text { b) } \mathrm{W}_{0} / \mathrm{L}_{\mathrm{C}}=0.25 ; \text { c) } \mathrm{W}_{0} / \mathrm{L}_{\mathrm{C}}=1 / 8 ; \text { d) } \mathrm{W}_{0} / \mathrm{L}_{\mathrm{C}}=1 / 15 ; \\
\sigma_{\mathrm{S}}=0.1: & \text { e) } \left.\mathrm{W}_{0} / \mathrm{L}_{\mathrm{C}}=0.5 ; \text { f) } \mathrm{W}_{0} / \mathrm{L}_{\mathrm{C}}=0.25 ; \mathrm{g}\right) \mathrm{W}_{0} / \mathrm{L}_{\mathrm{C}}=1 / 8 ; \text { h) } \mathrm{W}_{0} / \mathrm{L}_{\mathrm{C}}=1 / 15
\end{array}
$$




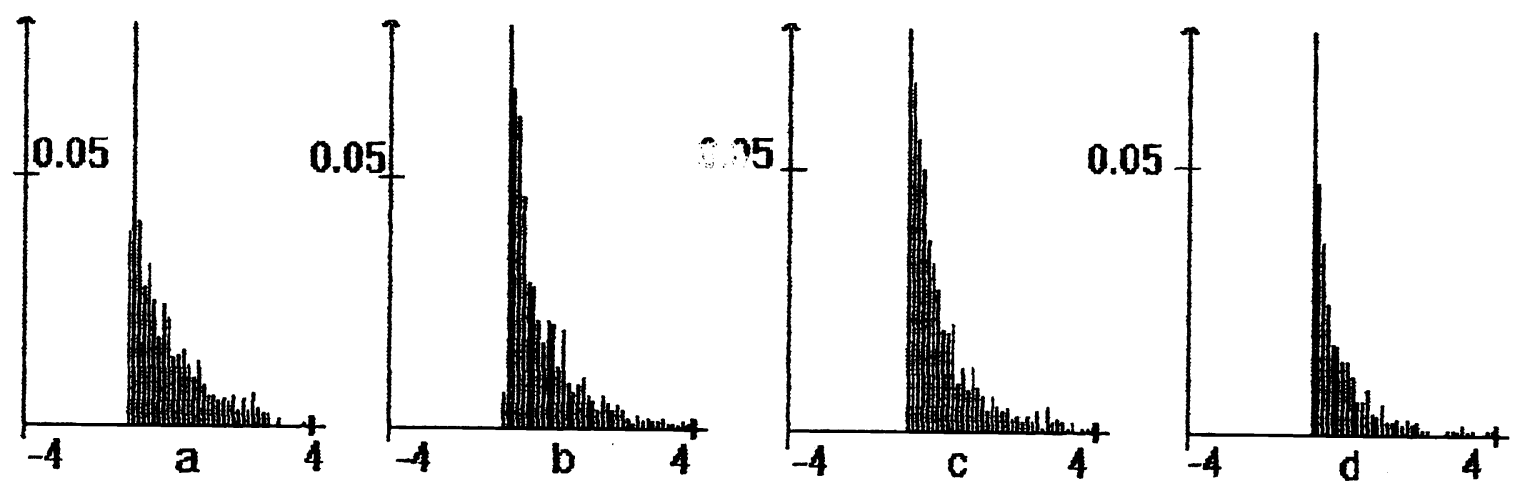

Fig.2. Histograms for probability density function of scattered intensity fluctuations at focused speckles diffraction from a rough surface. Values of intensity are normalized to that ones with zero mean and the unit standard deviation. Case of 4 speckles focused on the rough surface. W is a radius of the focused spot, $\mathrm{W}=20 \lambda ; \mathrm{W} / \mathrm{L}_{\mathrm{C}}=2 ;$ a) $\left.\left.\sigma_{\mathrm{S}}=0.001 ; \mathrm{b}\right) \sigma_{\mathrm{S}}=0.08 ; \mathrm{c}\right) \sigma_{\mathrm{S}}=0.1 ;$ d) $\sigma_{\mathrm{S}}=0.2$

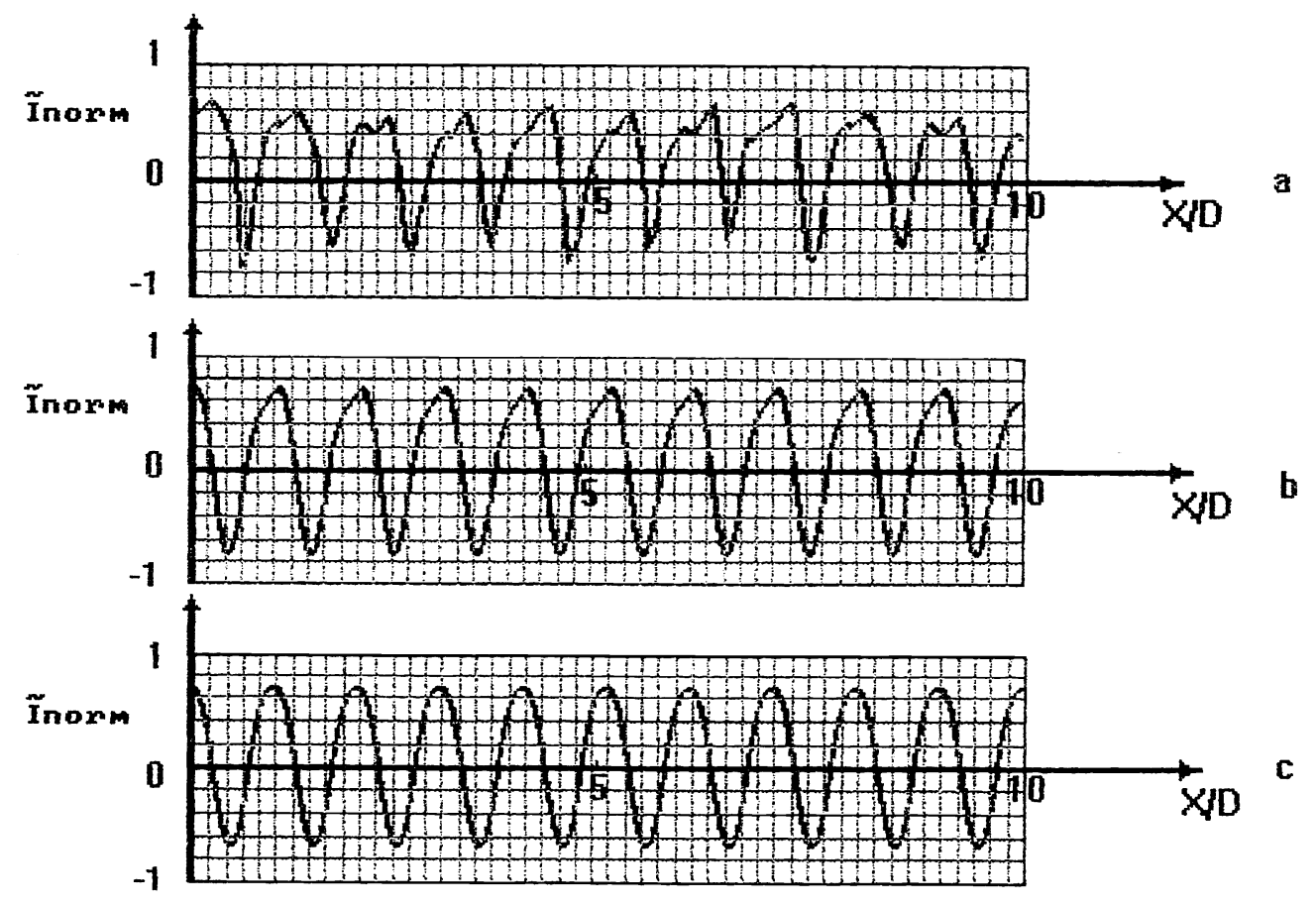

Fig. 3. The plot of dependence of alternate component of temporal intensity fluctuation of a scattered field (normalized on a maximal value) on waist beam center coordinate. The beam scans the surface having harmonic relief. 


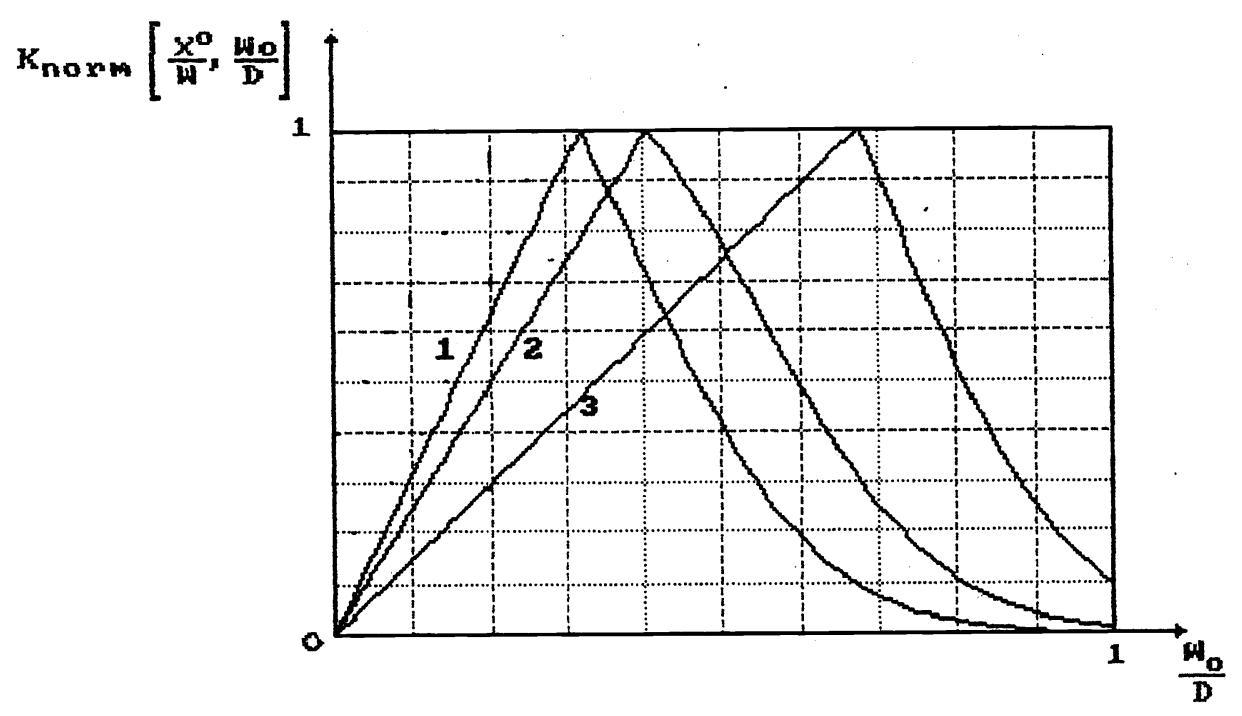

Fig. 4. Amplitude-frequency characteristics.

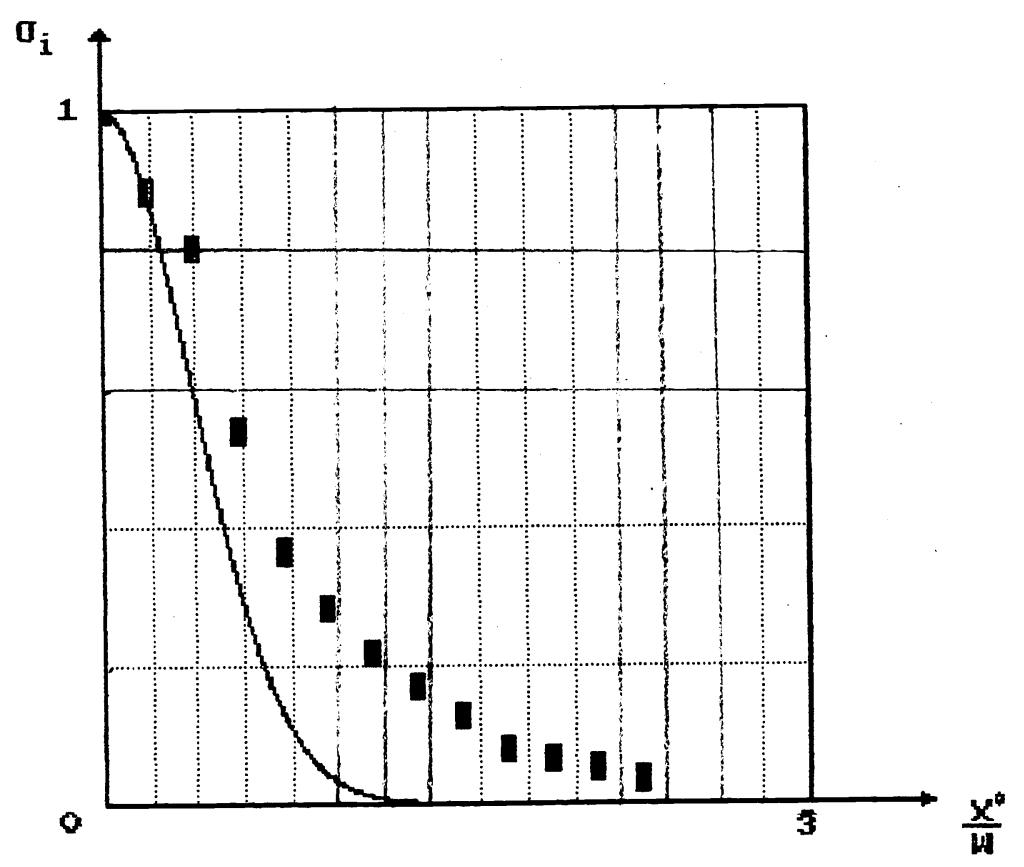

Fig. 5. Dependence of $\sigma_{\mathrm{I}}$ on observation point coordinate. 


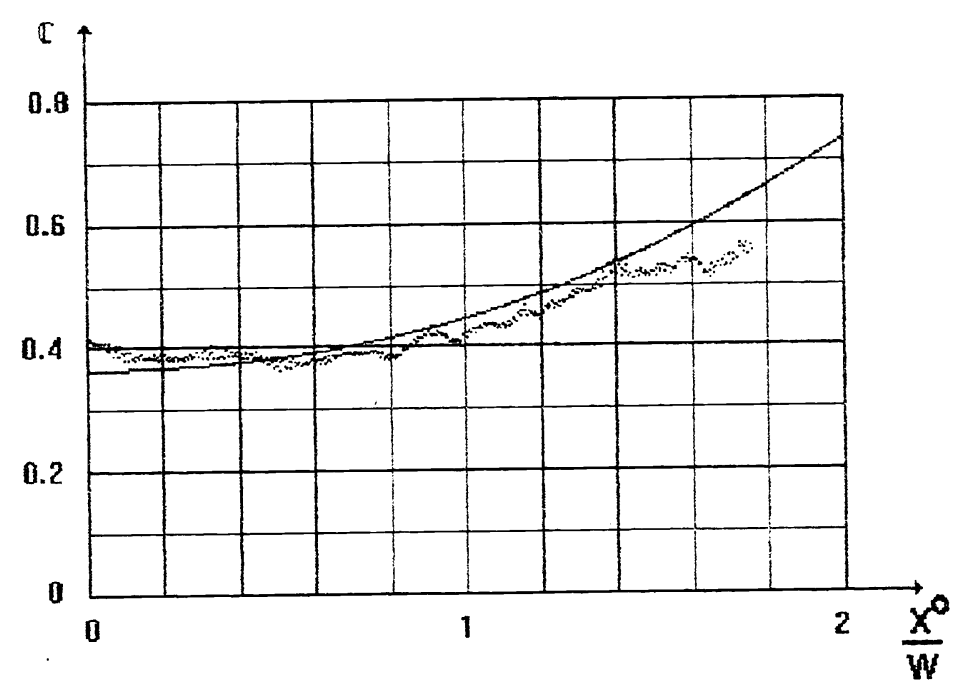

Fig. 6. Dependence of speckle contrast on observation point coordinate. 